\title{
Metallography of Powder Metallurgy Materials
}

\begin{abstract}
Alan Lawley* and Thomas F. Murphy**
The utility of metallography in understanding and applying processingmicrostructure-property relations in conventional cast or wrought metallic materials is well established. Powder metallurgy is now a mature commercial metalforming technology which frequently introduces an important variable in terms of the microstructure of the material, namely porosity. This presentation will discuss implications of the presence of pores in metallographic laboratory techniques and present case studies to illustrate how metallography has been instrumental in advancing understanding of the processing-microstructureproperty relation and failure in a range of powder metallurgy and particulate materials.
\end{abstract}

Metallographic preparation of powder metallurgy $(\mathrm{P} / \mathrm{M})$ components presents a number of challenges, the most obvious being the accurate portrayal of the porosity, both as an interconnected network and as individual sections through the network. Grinding and polishing, and even sectioning in some cases, require a great deal of care. Alteration of the pore morphology or not revealing open pores is of great concern. Additionally, the methods used to add the alloying elements often result in a heterogeneous distribution of the additives and consequently, complex microstructures containing a variety of transformation products. Traditional etching techniques many times are not effective for P/M materials. New or modified chemical etchants or stains are sometimes required to reveal the microstructure. Techniques developed to address these challenges intrinsic to powder metallurgy materials will be discussed.

The utility of optical microscopy, in combination with scanning electron microscopy, energy dispersive x-ray analysis and element mapping, is illustrated via application to the identification of fatigue failure in a P/M precipitation hardened stainless steel coated with tungsten carbide. Metallography identified the origin of the problem as breakdown of the carbide coating. Free carbon diffused into the stainless steel and reacted with the chromium to form chromium carbide at grain boundaries, leading to intergranular fracture.

Scanning electron fractography identified the cause of failure of a pressed and sintered ferrous oil pump gear with a density of about $87 \%$ theoretical density. Multiple fatigue cracks caused by excessive stress in the body of the spline were responsible for the service failure. The problem was alleviated by induction heat treatment of the bore spline to increase its fatigue strength.

Porous powder metallurgy parts are frequently joined using a sinter brazing cycle where the individual parts are both sintered and joined during the same furnace operation. Service failures, in the form of cracks adjacent to the joint, were experienced in an assembly where two P/M parts of different chemical 
composition were sinterbrazed. It was observed that all the cracks were seen along the same side of the joint. A combination of light and scanning electron microscopy, including both energy dispersive x-ray analysis and image analysis, showed the cause of the failures to be a combination of high carbon from one part and increased alloying from the brazing material resulting in an excessive amount of retained austenite. The retained austenite transformed to brittle untempered martensite and cracked under the service load. Changing the chemical composition of the suspect part to one lower in carbon content eliminated the problem.

Quantifying the degree of sinter in ferrous P/M materials has been, at best, subjective. A method is described which addresses this problem by application of the box counting technique. Digital representations of pore perimeters are obtained from optical micrographs. Log-log plots of the box size and box counts produce a linear relationship in which the negative of the slope of the line is the box count dimension. The lower the slope, the fewer the boxes counted and consequently, the better is the degree of sinter.

Porous metal filters used to remove unwanted particulate matter from gasses and liquids are low-density sintered P/M parts. They are formed using compaction pressures substantially lower than that used in the manufacture of conventional P/M parts. The resulting compacts contain a large volume percent porosity with the pore size distribution controlled by the combination of the density and particle size of the base powder. In this case, metallography is used as a tool to assist the part designer in determining the correct combination of density, base metal particle size, and processing conditions required to form a filter capable of trapping target particles from the fluid or gas. The metallographic specimen is examined using an automated image analysis system where the pore space is extracted from the grayscale image. The image of the pores is processed and a circular particle shape fit into the pores, starting with the largest. Iterations of this fitting are repeated using smaller and smaller circles. As a circle is placed into the porosity, it is subtracted from the total pore space. The remaining pore space is used for fitting of the smaller circles. Also, as the large spaces are filled, smaller and smaller particles are capable of being trapped in the remaining space. This process continues until the smallest circles representing trapped particles are lowest in importance to the filter. At the completion of the process, a predicted particle size distribution can be measured from the circles inserted into the pore space. This distribution can be determined on filters made using different base materials and/or processing routes.

\author{
*Grosvenor Professor \\ Drexel University \\ Philadelphia, PA
}

\author{
**Scientist, Laboratory Services \\ Hoeganaes Corporation \\ Cinnaminson, NJ
}

